\title{
Comparing the clinical characteristics and outcomes of COVID-19-associate pulmonary aspergillosis (CAPA): a systematic review and meta-analysis
}

\author{
Woon Hean Chong ${ }^{1}$ (D) Biplab K. Saha ${ }^{2} \cdot$ Kristoffer P. Neu $^{3}$
}

Received: 21 April 2021 / Accepted: 14 September 2021 / Published online: 27 September 2021

(c) Springer-Verlag GmbH Germany, part of Springer Nature 2021

\begin{abstract}
Purpose Invasive pulmonary aspergillosis has been increasingly recognized in COVID-19 patients, termed COVID-19-associate pulmonary aspergillosis (CAPA). Our meta-analysis aims to assess the clinical characteristics and outcomes of patients diagnosed with CAPA compared to those without CAPA.

Methods We searched the Pubmed, Cochrane Library, SCOPUS, and Web of Science databases for studies published between January 1, 2020 and August 1, 2021, containing comparative data of patients diagnosed with CAPA and those without CAPA. Results Eight cohort studies involving 729 critically ill COVID-19 patients with comparative data were included. CAPA patients were older (mean age 66.58 vs. 59.25 years; $P=0.007$ ) and had underlying chronic obstructive pulmonary disease (COPD) (13.7 vs. 6.1\%; OR 2.75; $P=0.05$ ). No differences in gender, body mass index (BMI), and comorbidities of diabetes and cancer were observed. CAPA patients were more likely to receive long-term corticosteroid treatment (15.0 vs. 5.3\%; OR $3.53 ; P=0.03$ ). CAPA patients had greater severity of illness based on sequential organ failure assessment (SOFA) score with a higher all-cause in-hospital mortality rate $(42.6$ vs. $26.5 \%$; OR $3.39 ; P<0.001)$ and earlier ICU admission from illness onset (mean 11.00 vs. 12.00 days; $P=0.003$ ). ICU length of stay (LOS), invasive mechanical ventilation (IMV) duration, the requirement of inotropic support and renal replacement therapy were comparable between the two groups.

Conclusions CAPA patients are typically older with underlying COPD and received long-term corticosteroid treatment. Furthermore, CAPA is associated with higher SOFA scores, mortality, and earlier onset of ICU admission from illness onset.
\end{abstract}

Keywords Coronavirus disease 2019 COVID-19 · COVID-19-associated pulmonary aspergillosis · CAPA · Invasive pulmonary aspergillosis $\cdot$ Risk factors $\cdot$ Outcomes

\section{Introduction}

Since coronavirus disease 2019 (COVID-19) was first recognized in early December 2019, it has resulted in the ongoing worldwide pandemic. COVID-19 is known to have a wide variety of clinical manifestations, from a mild flu-like

Woon Hean Chong

keenan_chong_woon_hean@nuhs.edu.sg

1 Department of Pulmonary and Critical Care Medicine, Albany Medical Center, 43 New Scotland Avenue, Albany, NY 12208, USA

2 Department of Pulmonary and Critical Care, Ozarks Medical Center, West Plains, MO 65775, USA

3 Department of Pulmonary and Critical Care, Albany Stratton VA Medical Center, Albany, NY 12208, USA illness to severe respiratory failure in the setting of COVID19 pneumonia-induced acute respiratory distress syndrome (ARDS). As the wave of COVID-19 continues to spread across the globe, secondary pulmonary infections from bacterial and fungal microorganisms have been increasingly recognized in hospitalized COVID-19 patients, associated with poor outcomes of increased mechanical ventilation requirement, multi-organ dysfunction, and mortality [1,2]. The diagnosis of invasive pulmonary aspergillosis is made based on the positive results of lower respiratory tract (LRT) cultures or polymerase chain reaction (PCR) for Aspergillus species, or using galactomannan (GM) biomarker obtained from serum or bronchoalveolar lavage (BAL) in COVID-19 patients, termed COVID-19-associated pulmonary aspergillosis (CAPA) [3-6]. Multiple diagnostic criteria have been used to define and identify patients with CAPA across many observational studies [7]. Although the incidence of fungal 
infection is $6.3 \%$ in hospitalized COVID-19 patients and is frequently seen in those who are critically ill, the actual risk factors and outcomes of CAPA remain unknown [1]. Our systematic review and meta-analysis aim to examine and discuss the clinical characteristics, risk factors, and outcome of COVID-19 patients diagnosed with CAPA compared to those without CAPA.

\section{Methods}

This systematic review was conducted and presented in accordance with Preferred Reporting Items for Systematic Reviews and Meta-Analyses (PRISMA) guidelines. Ethical approval and informed consent were not required for this study as it was a systematic review of previously published studies. The protocol for this review was registered and published in the International Prospective Register of Systematic Reviews (PROSPERO) under reference number CRD42021247177.

\section{Search criteria and selection}

A literature search was performed through Pubmed, Cochrane Library, SCOPUS, and Web of Science databases for articles published, using the keywords and respective Medical Subjects Headings (MeSH) terms: ("coronavirus disease 2019" OR "COVID-19" OR "severe acute respiratory syndrome coronavirus 2" OR "SARS-CoV-2") AND ("COVID-19-associated pulmonary aspergillosis" OR "CAPA" OR "fungal infections" OR "secondary infections" OR "fungal pneumonia" OR "mycosis" OR "Aspergillosis" OR "Aspergillus" OR "invasive pulmonary aspergillosis" OR "IPA") OR ("risk factors" OR "prognostic factors" OR "mortality factors") OR ("mortality" OR "Outcomes"). Moreover, to detect additional studies, any cited references were reviewed to identify relevant literature that met our inclusion criteria.

\section{Inclusion criteria}

We included studies that: (1) contains comparative data describing the clinical characteristics, risk factors, and outcomes of hospitalized COVID-19 adults with CAPA and those without CAPA; (2) the diagnosis of COVID-19 was made by reverse transcriptase-polymerase chain reaction (RT-PCR) in all cases from respiratory tract specimens that include nasal and pharyngeal swabs, sputum, tracheal aspirate (TA), non-directed bronchial lavage (NBL), and BAL; (3) were published between January 1, 2020 and August 1, 2021 in peer-review journals; and (4) in which CAPA diagnosis was made using recognized invasive pulmonary aspergillus classifications such as: (1) Modified AspICU[8],
(2) CAPA Consensus definition[9], (3) European Organization for Research and Treatment of Cancer and Mycoses Study Group Education and Research Consortium (EORTC/ MSG)[10], and (4) European Excellence Center for Medical Mycology and International Society of Human and Animal Mycology (ECMM/ISHAM) consensus criteria[5].

\section{Exclusion criteria}

We excluded studies that: (1) describe Aspergillus colonization from the LRT specimens (defined as BAL or NBL) or if Aspergillus species was isolated from LRT specimens, but the authors did not specify whether these represented invasive pulmonary aspergillosis or colonization, and the data provided was inadequate to make any distinction. Aspergillus species identified on sputum or TA will be classified as colonization; (2) systematic reviews, literature reviews, editorials, conference abstracts, opinion articles, meta-analysis, case reports, or studies containing less than 30 patients (defined as case series); (3) studies involving non-adult COVID-19 patients (less than 18 years of age); (4) studies that did not have comparative data between CAPA and non-CAPA patients (controls); (5) studies where pulmonary aspergillosis was concurrently diagnosed with other microorganisms such as bacterial, viral, or non-Aspergillus fungal microorganisms from similar respiratory tract cultures; (6) articles describing aspergillosis obtained from non-respiratory tract cultures; (7) studies published in languages other than English were excluded if no translated version of the manuscript was available; and (8) studies in which the diagnosis of CAPA was made during postmortem examination.

\section{Data collection and synthesis}

Two researchers (W.C. and B.S.) independently screened the titles and abstracts, and reviewed the full texts of articles to identify studies that compare the clinical characteristics, risk factors, and outcomes of hospitalized COVID-19 patients diagnosed with CAPA. Any disagreements were resolved by discussion or third researcher (K.N.). The extracted data from full texts of included studies was added into a standardized Excel (Microsoft Corporation) form. The following information was collected and summarized in Tables 1 and 2 for each group of patients involving study characteristics, clinical characteristics, risk factors, and associated outcomes. The definitions of CAPA.

\section{Outcomes}

The primary outcome was the clinical characteristics and risk factors for developing CAPA in COVID-19 patients compared to those without CAPA diagnosis. The secondary outcome was the outcomes of all-cause in-hospital mortality, 


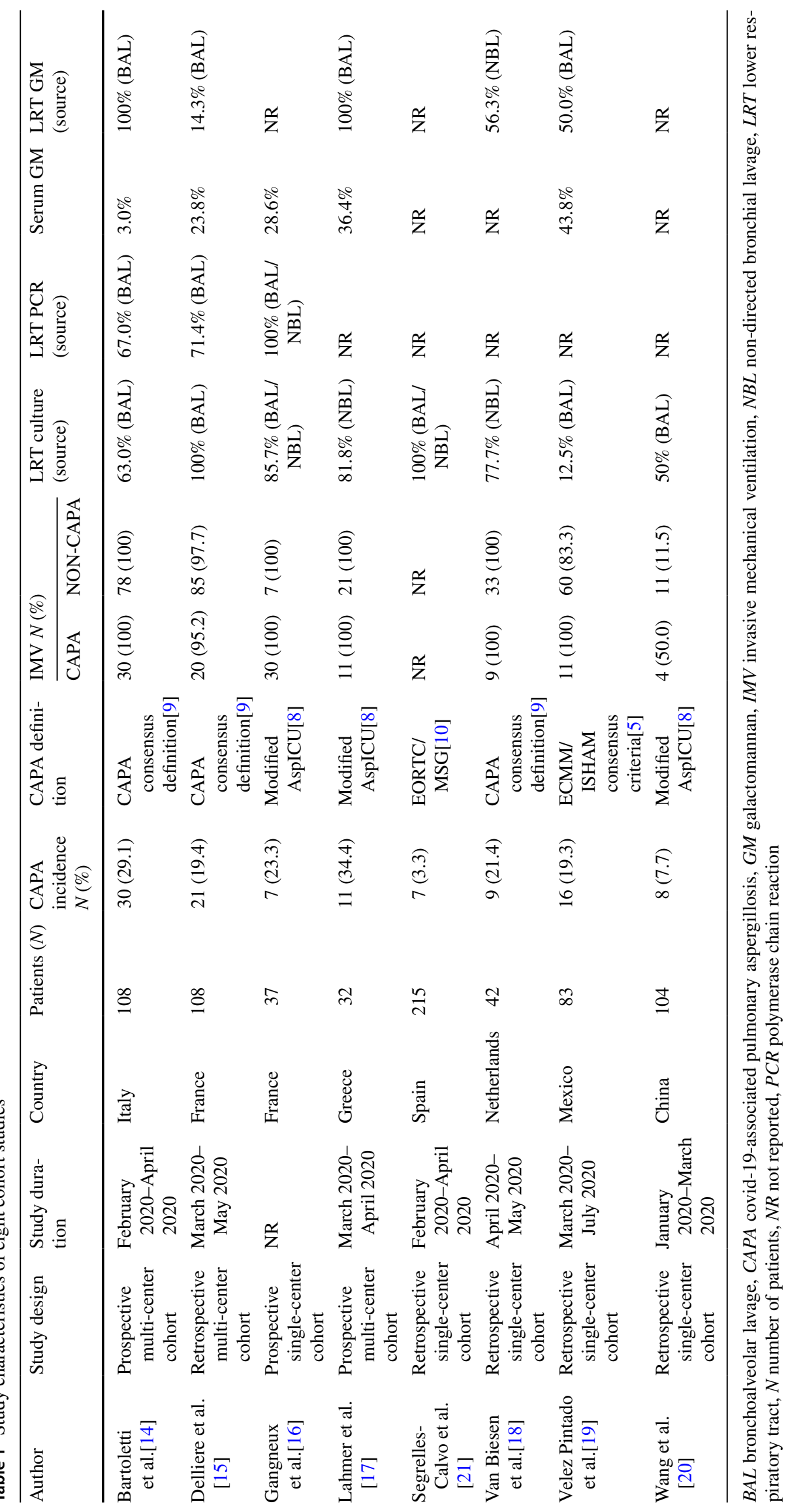


Table 2 Comparative data on the clinical characteristics, risk factors, and outcomes of COVID-19 patient diagnosed with and without CAPA diagnosis

\begin{tabular}{|c|c|c|c|c|c|c|c|}
\hline Variables & Studies $(N)$ & CAPA & No CAPA & OR/MD & $95 \% \mathrm{CI}$ & $P$ value & $\begin{array}{l}\text { Hetero- } \\
\text { geneity } \\
\left(\mathrm{I}^{2}\right)\end{array}$ \\
\hline \multicolumn{8}{|l|}{ Clinical characteristics } \\
\hline Age (Y) Mean \pm S.D & 8 & $66.58 \pm 4.55$ & $59.25 \pm 3.42$ & MD 7.52 & $2.02-13.03$ & 0.007 & $74 \%$ \\
\hline Male & 7 & $72.5 \%(74 / 102)$ & $70.6 \%(291 / 412)$ & OR 0.82 & $0.43-1.55$ & 0.54 & $27 \%$ \\
\hline BMI $\left(\mathrm{kg} / \mathrm{m}^{2}\right)$ Mean \pm S.D & 4 & $27.80 \pm 1.71$ & $27.88 \pm 0.74$ & $\mathrm{MD}-0.46$ & {$[-1.93,1.02]$} & 0.54 & $46 \%$ \\
\hline \multicolumn{8}{|l|}{ Comorbidities } \\
\hline COPD & 7 & $13.7 \%(14 / 102)$ & $6.1 \%(25 / 412)$ & OR 2.75 & $1.00-7.52$ & 0.05 & $38 \%$ \\
\hline Diabetes & 7 & $26.5 \%(27 / 102)$ & $23.3 \%(94 / 404)$ & OR 1.20 & $0.71-2.01$ & 0.49 & $0 \%$ \\
\hline Cancer & 4 & $8.2 \%(5 / 61)$ & $3.7 \%(10 / 271)$ & OR 2.25 & $0.68-5.07$ & 0.14 & $0 \%$ \\
\hline \multicolumn{8}{|l|}{ Long-term medications } \\
\hline Long-term corticosteroid & 3 & $15.0 \%(9 / 60)$ & $5.3 \%(10 / 190)$ & OR 3.53 & $1.16-10.69$ & 0.03 & $9 \%$ \\
\hline Long-term immunosuppressants & 2 & $10.0 \%(3 / 30)$ & $7.1 \%(8 / 112)$ & OR 1.87 & $0.28-12.29$ & 0.52 & $25 \%$ \\
\hline \multicolumn{8}{|l|}{ COVID-19 therapies } \\
\hline Initial antibiotic treatment & 5 & $82.5 \%(52 / 63)$ & $81.6 \%(391 / 479)$ & OR 0.88 & $0.39-1.97$ & 0.75 & $12 \%$ \\
\hline Initial corticosteroid treatment & 4 & $49.2 \%(30 / 61)$ & $66.8 \%(300 / 449)$ & OR 0.69 & $0.19-2.58$ & 0.58 & $73 \%$ \\
\hline Tocilizumab & 4 & $55.4 \%(41 / 74)$ & $38.9 \%(171 / 440)$ & OR 1.85 & $0.88-3.89$ & 0.10 & $18 \%$ \\
\hline Hydroxychloroquine & 4 & $70.3 \%(52 / 74)$ & $81.6 \%(359 / 440)$ & OR 0.43 & $0.07-2.68$ & 0.36 & $84 \%$ \\
\hline \multicolumn{8}{|l|}{ ICU A = admission } \\
\hline $\begin{array}{l}\text { Illness onset to ICU admission } \\
\text { (D) mean } \pm \text { S.D }\end{array}$ & 2 & $11.00 \pm 2.50$ & $12.00 \pm 3.00$ & $\mathrm{MD}-1.00$ & {$[-1.66,-0.34]$} & 0.003 & $0 \%$ \\
\hline SOFA Score mean \pm S.D & 3 & $9.37 \pm 2.02$ & $7.27 \pm 1.32$ & MD 2.57 & $1.46-3.68$ & $<0.001$ & $0 \%$ \\
\hline \multicolumn{8}{|l|}{ Outcomes } \\
\hline Mortality & 7 & $42.6 \%(43 / 101)$ & $26.5 \%(139 / 524)$ & OR 3.39 & $1.97-5.86$ & $<0.001$ & $0 \%$ \\
\hline ICU LOS (D) mean \pm S.D & 6 & $25.72 \pm 7.19$ & $18.44 \pm 4.06$ & MD 6.85 & {$[-2.08,15.79]$} & 0.13 & $84 \%$ \\
\hline IMV duration (D) mean \pm S.D & 3 & $17.00 \pm 2.94$ & $16.00 \pm 0.82$ & $\mathrm{MD}-1.66$ & {$[-5.49,2.16]$} & 0.39 & $0 \%$ \\
\hline RRT & 4 & $37.1 \%(26 / 70)$ & $19.1 \%(54 / 282)$ & OR 2.30 & $0.95-5.57$ & 0.06 & $42 \%$ \\
\hline Inotropic support & 3 & $79.0 \%(49 / 62)$ & $75.8 \%(141 / 186)$ & OR 1.19 & $0.56-2.56$ & 0.65 & $0 \%$ \\
\hline
\end{tabular}

$B M I$ body mass index, $C A P A$ covid-19-associated pulmonary aspergillosis, $C I$ confidence intervals, $C O P D$ chronic obstructive pulmonary disease, $D$ days, $I M V$ invasive mechanical ventilation, $L O S$ length of stay, $N$ number of patients, $M D$ mean difference, $N R$ not reported, $O R$ odd ratio, $R R T$ renal replacement therapy, $S D$ standard deviation, $S O F A$ sequential organ failure assessment, $Y$ years

ICU length of stay (LOS), invasive mechanical ventilation (IMV) duration, inotropic and renal replacement therapy (RRT) requirement among COVID-19 patients with CAPA.

\section{Quality assessment}

Two researchers (W.C. and B.S.) performed quality assessments using the Newcastle-Ottawa Scale (NOS), containing nine items, for the cohort studies. Briefly, the NOS scale assesses three important features of the study such as: adequacy of the selection of exposed and non-exposed cohorts, the comparability of groups, and the adequacy of outcomes assessment with a total score ranging from 0 to 9 [11]. The study quality can be divided into three groups: Low quality " $0-3$ ", moderate quality " $4-6$ ", and high quality "7-9" (Table 3). During the quality assessment of the included studies, any disagreements were resolved by discussion.

\section{Statistical analysis}

A meta-analysis was performed for the primary and secondary outcomes using the Review Manager (RevMan) software, Version 5.4, The Cochrane Collaboration, 2020. Dichotomous outcomes were assessed using Mantel-Haenszel statistical method and measured in odds ratios (ORs) and their $95 \%$ confidence intervals (CIs). Continuous outcomes were evaluated by inverse variance method and measured in mean difference (MDs). Using DerSimonian and Laird's random-effects model, pooled ORs, MDs, and 95\% CIs were calculated, and extracted outcomes were pooled by weighted averages [12]. The random-effects model was preferred over the fixed-effects model as we suspected that clinical heterogeneity might be present due to the variability across the included studies regarding differences in clinical practices, patient population characteristics, and 
Table 3 The table shows the results of the Newcastle-Ottawa Scale (NOS) [11] performed for eight cohort studies

\begin{tabular}{|c|c|c|c|c|c|c|c|c|c|c|}
\hline \multirow[t]{2}{*}{ Author(s) } & \multirow[t]{2}{*}{ Cohort studies } & \multicolumn{4}{|c|}{ Selection } & \multirow{2}{*}{$\begin{array}{l}\text { Comparability } \\
(* *)\end{array}$} & \multicolumn{3}{|c|}{$\begin{array}{l}\text { Outcome/ } \\
\text { exposure }\end{array}$} & \multirow[t]{2}{*}{$\begin{array}{l}\text { Total of } \\
9 \text { scores }\end{array}$} \\
\hline & & 1 & 2 & 3 & 4 & & a & $\mathrm{b}$ & $\mathrm{c}$ & \\
\hline Bartoletti et al. [14] & Prospective cohort & $*$ & $*$ & $*$ & $*$ & $* *$ & $*$ & $*$ & $*$ & 9 \\
\hline Delliere et al. [15] & Retrospective cohort & $*$ & $*$ & $*$ & $*$ & $* *$ & * & $*$ & $*$ & 9 \\
\hline Gangneux et al. [16] & Prospective cohort & $*$ & $*$ & $*$ & $*$ & $* *$ & $*$ & $*$ & $*$ & 9 \\
\hline Lahmer et al.[17] & Prospective cohort & * & $*$ & $*$ & $*$ & $* *$ & $*$ & $*$ & * & 9 \\
\hline Segrelles-Calvo et al. [21] & Retrospective cohort & $*$ & $*$ & $*$ & $*$ & $*$ & * & $*$ & $*$ & 8 \\
\hline Van Biesen et al. [18] & Retrospective cohort & $*$ & $*$ & $*$ & $*$ & $* *$ & $*$ & $*$ & $*$ & 9 \\
\hline Velez Pintado et al. [19] & Retrospective cohort & $*$ & $*$ & $*$ & $*$ & $* *$ & $*$ & $*$ & $*$ & 9 \\
\hline Wang et al. [20] & Retrospective cohort & $*$ & $*$ & * & $*$ & $* *$ & $*$ & $*$ & $*$ & 9 \\
\hline
\end{tabular}

(1) Representatives of the exposed cohorts

(2) Selection of the non-exposed cohorts

(3) Ascertainment of exposure

(4) The outcome of interest was not present at the start of the study

Comparability: study controls were compared for the most important factor and additional factors

(a) Assessment of the outcome

(b) Enough follow-up for the outcome

(c) Adequacy of follow-up

*Positive variable diagnostic criteria for CAPA diagnosis. Furthermore, we aimed to assess the mean distribution of results across the eight studies with various sample sizes without disregarding the results of small studies and giving extra weightage to results from larger studies. Statistical heterogeneity among studies was assessed by the $\mathrm{I}^{2}$ statistic. High heterogeneity being classified as $\mathrm{I}^{2}$ statistics of 50 and greater, and low was with $\mathrm{I}^{2}$ statistics less than 50\% [13]. A $P$ value of $<0.05$ was considered statistically significant. Publication bias was assessed by visual inspection of the funnel plot.

\section{Results}

\section{Study selection and characteristics}

565 studies were identified during the initial search. After removing duplicates and those not meeting the inclusion criteria (by title, abstract, and full text), 8 eligible observational studies were included in this meta-analysis (Fig. 1). The study characteristics of the 8 studies included were described in Table 1. All observational studies were cohort studies. Among the 8 cohorts, $62.5 \%$ (5/8) were retrospective, and the remainder were prospectively designed studies. $62.5 \%$ (5/8) were single-center studies, and $37.5 \%(3 / 8)$ were multi-center studies. The majority $(75 \%[6 / 8])$ of the studies were published from Europe (France, Greece, Italy, Netherlands, and Spain), and the remainder from China and Mexico. The summary of the study qualities was shown in Table $3.87 .5 \%$ (7/8) of the studies had the maximum scores of nine [14-20], and the remaining study by Segrelles-Calvo et al. had a score of eight [21].

A total of 729 COVID-19 patients were included, in which $14.9 \%$ (109/729) were diagnosed with CAPA, with incidence ranging between 3.3 and $34.4 \%$. All observational studies were conducted during the first wave of the COVID-19 pandemic ranging from January 2020 to July 2020. An overwhelming majority of COVID-19 patients with and without CAPA diagnosis (Table 1) were critically ill and required IMV. The mycological evidence for CAPA were based on LRT cultures in 67.8\% (74/109) of patients followed by BAL GM [52.3\% (57/109)], LRT PCR [38.5\% (42/109)], and serum GM [17.4\% (19/109)].

\section{Clinical characteristics}

COVID-19 patients diagnosed with CAPA were typically older (mean age 66.58 vs. 59.25 years; MD 7.52; 95\% CI $\left.2.02-13.03 ; \mathrm{I}^{2}=74 \% ; P=0.007\right)$ than those without CAPA (Table 2 and Fig. 2). No gender (male 72.5 vs. $70.6 \%$; OR $\left.0.82 ; 95 \% \mathrm{CI} 0.43-1.55 ; \mathrm{I}^{2}=27 \% ; P=0.54\right)$ and body mass index (BMI) (mean 27.80 vs. $27.88 \mathrm{~kg} / \mathrm{m}^{2}$; MD - 0.46; 95\% CI $\left.[-1.93,1.02] ; \mathrm{I}^{2}=46 \% ; P=0.54\right)$ difference was observed in patients with and without CAPA diagnosis (Figs. 2 and 3).

\section{Comorbidities}

CAPA patients were more likely to have chronic obstructive pulmonary disease (COPD) (13.7 vs. 6.1\%; OR 2.75; 


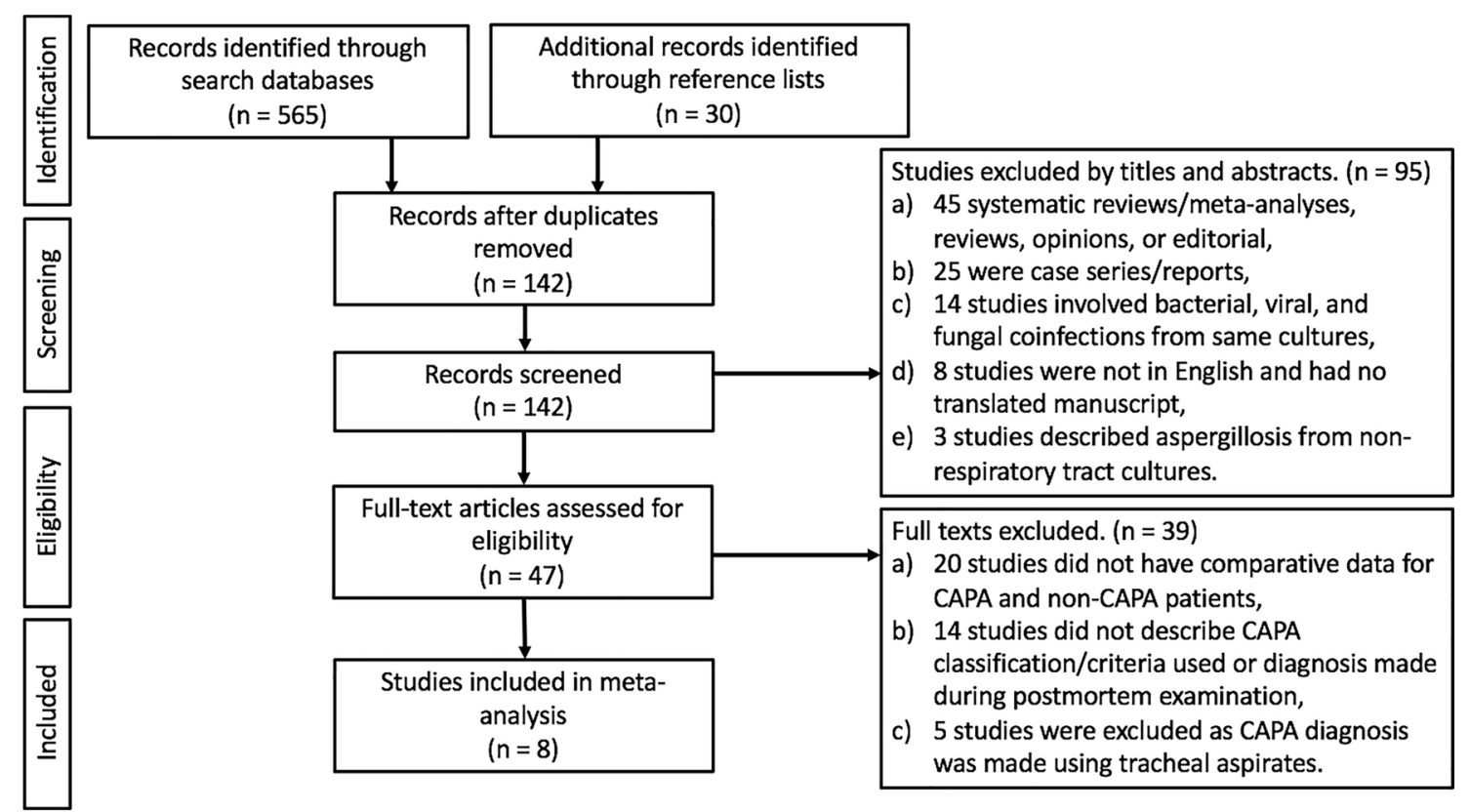

Fig. 1 Flow diagram of study selection

95\% CI $\left.1.00-7.52 ; \mathrm{I}^{2}=38 \% ; P=0.05\right)$ than those without CAPA (Table 2 and Fig. 3). Other comorbidities such as diabetes (26.5 vs. 23.3\%; OR 1.20 ; $95 \%$ CI $0.71-2.01$; $\left.\mathrm{I}^{2}=0 \% ; P=0.49\right)$ and cancer $(8.2$ vs. $3.7 \%$; OR $2.25 ; 95 \%$ CI $\left.0.68-5.07 ; \mathrm{I}^{2}=0 \% ; P=0.14\right)$ were similar between the two groups (Fig. 3).

\section{Long-term medications and COVID-19 therapies}

A greater proportion of CAPA patients were requiring longterm corticosteroid treatment (15.0 vs. $5.3 \%$; OR 3.53 ; $95 \%$ CI $\left.1.16-10.69 ; \mathrm{I}^{2}=9 \% ; P=0.03\right)$ than those without CAPA (Table 2 and Fig. 3). However, the type, doses, frequency, and duration of corticosteroids administration were not well described among the three studies included. Two studies described COVID-19 patients requiring long-term immunosuppressants other than corticosteroids but no difference in the risk of developing CAPA (10.0 vs. 7.1\%; OR 1.87; 95\% CI $0.28-12.29 ; \mathrm{I}^{2}=25 \% ; P=0.52$ ) was observed (Fig. 3) $[15,18]$. Furthermore, the type, frequency, and duration of immunosuppressants were not well described.

In terms of COVID-19 therapies, initial corticosteroids (49.2 vs. $66.8 \%$; OR $0.69 ; 95 \%$ CI $0.19-2.58 ; \mathrm{I}^{2}=73 \%$; $P=0.58)$ and tocilizumab (55.4 vs. $38.9 \%$; OR $1.85 ; 95 \%$ CI $\left.0.88-3.89 ; \mathrm{I}^{2}=18 \% ; P=0.10\right)$ therapies were not demonstrated to increase the likelihood of developing CAPA (Table 2 and Fig. 4). Similar results were seen when antibiotic (82.5 vs. $81.6 \%$; OR 0.88 ; $95 \%$ CI $0.39-1.97$;
$\left.\mathrm{I}^{2}=12 \% ; P=0.75\right)$ and hydroxychloroquine (70.3 vs. $81.6 \%$; OR 0.43 ; 95\% CI $0.07-2.68 ; \mathrm{I}^{2}=84 \% ; P=0.36$ ) therapies were initially given (Fig. 4).

\section{Severity of illness and outcomes}

COVID-19 patient with CAPA diagnosis were likely to have a higher severity of illness based on sequential organ failure assessment (SOFA) score (mean 9.37 vs. 7.27; MD 2.57; 95\% CI $\left.1.46-3.68 ; \mathrm{I}^{2}=0 \% ; P<0.001\right)$ with rapid deterioration of clinical status requiring earlier ICU admission from illness onset (mean 11.00 vs. 12.00 days; MD - 1.00; 95\% CI [ - 1.66, - 0.34]; $\mathrm{I}^{2}=0 \% ; P=0.003$ ) (Table 2 and Fig. 2).

The all-cause in-hospital mortality rate is greater in CAPA patients (42.6 vs. $26.5 \%$; OR 3.39 ; $95 \%$ CI $\left.1.97-5.86 ; \mathrm{I}^{2}=0 \% ; P<0.001\right)$ compared to those without CAPA (Table 2 and Fig. 4). However, ICU length of stay (LOS) (mean 25.72 vs. 18.44 days; MD 6.85; 95\% CI [ $-2.08,15.79] ; \mathrm{I}^{2}=84 \% ; P=0.13$ ) and IMV duration (mean 17.00 vs. 16.00 days; MD -1.66; 95\% CI [ - 5.49, $\left.2.16] ; \mathrm{I}^{2}=0 \% ; P=0.39\right)$ were comparable between the two groups (Fig. 2). The requirement of inotropic support (79.0 vs. $75.8 \%$; OR $1.19 ; 95 \%$ CI $0.56-2.56 ; \mathrm{I}^{2}=0 \% ; P=0.65$ ) and renal replacement therapy (RRT) (37.1 vs. $19.1 \%$; OR $2.30 ; 95 \%$ CI $\left.0.95-5.57 ; \mathrm{I}^{2}=42 \% ; P=0.06\right)$ were equal in those with and without CAPA diagnosis (Fig. 4). 


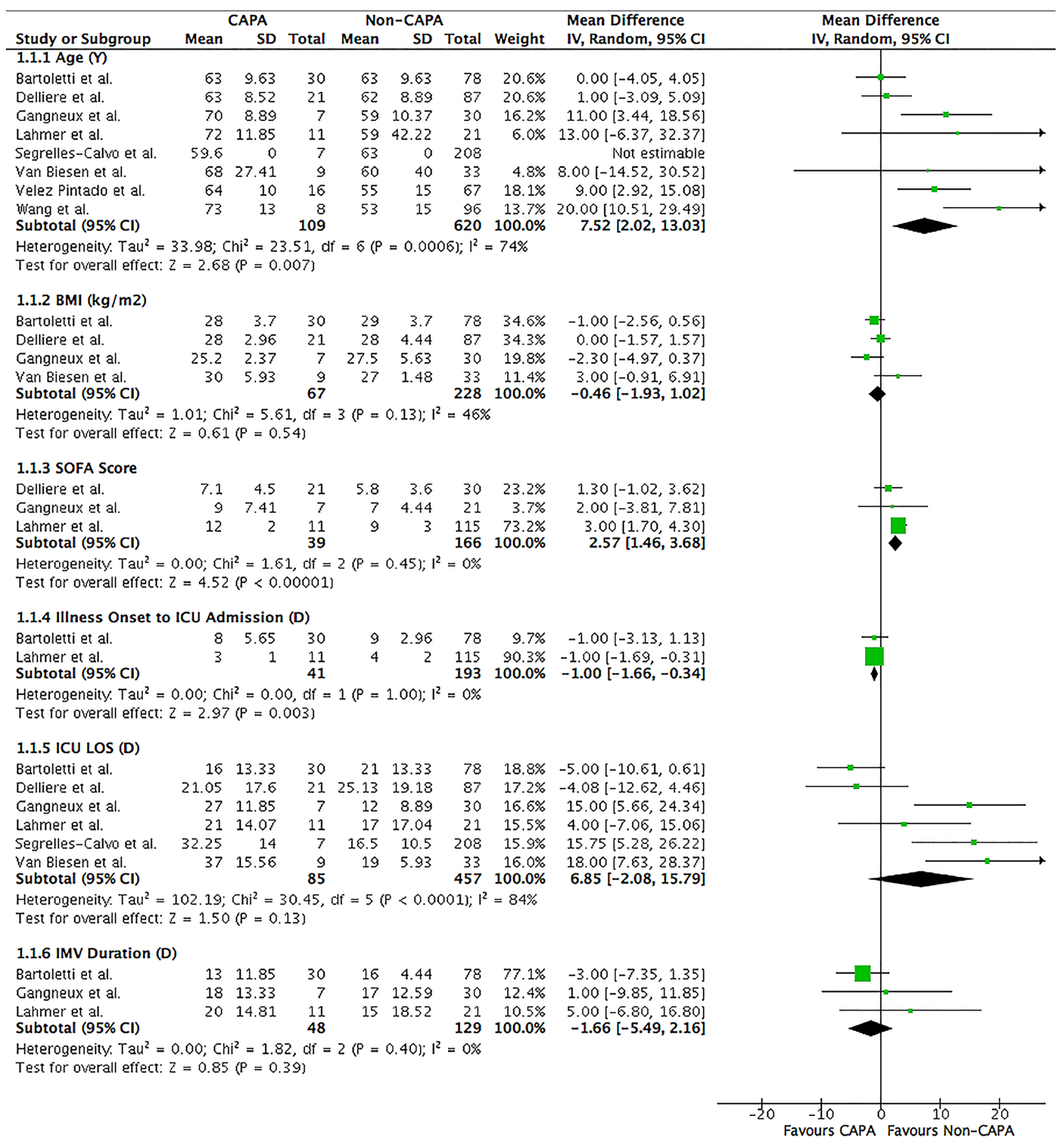

Fig. 2 Forrest plot of COVID-19 patients divided into CAPA versus non-CAPA. Clinical characteristics and risk factors of age, BMI, SOFA score, illness onset to ICU admission, ICU LOS, and IMV duration were assessed. Mean differences were calculated by the inverse variance statistical method with a random-effects model. $C I$

\section{Discussion}

Among the eight cohorts conducted during the first wave of the pandemic involving 729 COVID-19 patients, $14.9 \%$ were confidence intervals, $D$ days, $d f$ degree of freedom, $I C U$ intensive care unit, $I M V$ invasive mechanical ventilation, $I V$ inverse variance, $L O S$ length of stay, $S D$ standard deviation, SOFA sequential organ failure assessment, $Y$ years

diagnosed with CAPA. The majority of patients were critically ill and required IMV. COVID-19 patients with CAPA diagnosis were typically older and had underlying COPD. No differences in gender, BMI, and other comorbidities of 
$\begin{array}{cccc}\text { CAPA } & \text { Non-CAPA } & \text { Odds Ratio } \\ \text { Study or Subgroup } & \text { Events Total Events Total } & \text { Weight } & \mathrm{M}-\mathrm{H}, \text { Random, } 95 \% \mathrm{Cl}\end{array}$

Odds Ratio

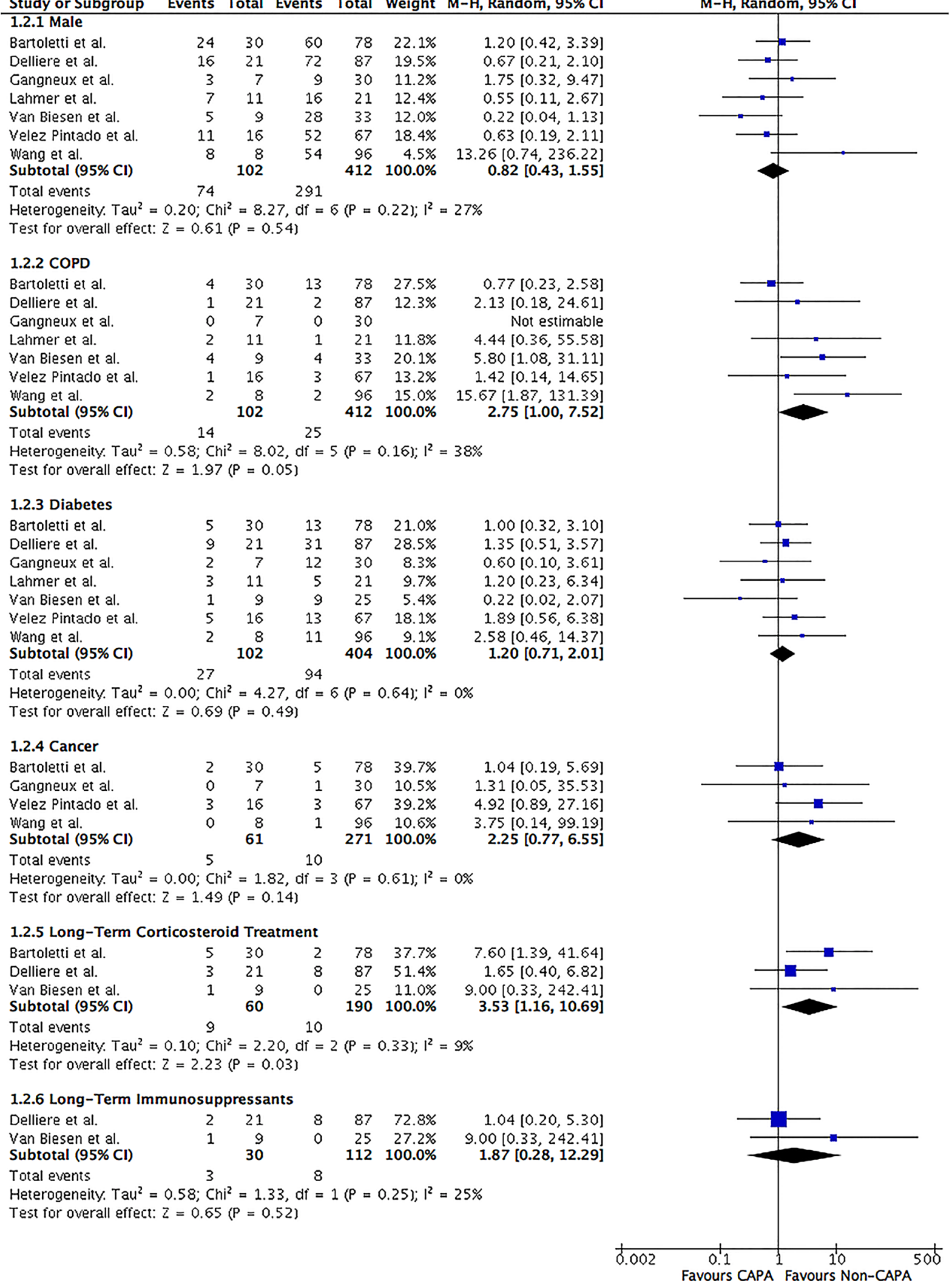


4Fig. 3 Forrest plot of COVID-19 patients divided into CAPA versus non-CAPA. Clinical characteristics and risk factors of male, COPD, diabetes, cancer, long-term corticosteroid treatment and immunosuppressants were assessed. The odds ratio was calculated by the Mantel-Haenszel method with a random-effects model. CI confidence intervals, $C O P D$ chronic obstructive pulmonary disease, $d f$ degree of freedom, $M-H$ Mantel-Haenszel

diabetes and cancer were observed. COVID-19 patients were more likely to require long-term corticosteroid treatment before CAPA diagnosis. Long-term use of immunosuppressants and COVID-19 therapies provided during the acute illness, such as corticosteroids, tocilizumab, antibiotics, and hydroxychloroquine, did not predispose to CAPA. COVID-19 patients diagnosed with CAPA were likely to have significant severity of illness based on SOFA score and earlier onset of ICU admission from illness onset with a higher all-cause in-hospital mortality rate. ICU LOS, IMV duration, the requirement of inotropic support and RRT were comparable between the two groups.

It is important to differentiate between colonization and true infection when diagnosing CAPA, as positive Aspergillus species identified on TA does not necessarily indicate CAPA and has not been shown to alter the clinical course and outcomes among critically ill COVID-19 patients [22-24]. In the setting of a compatible clinical presentation, positive TA should be an indication to perform a confirmatory test involving serum biomarkers or BAL for cultures, PCR, and GM when appropriate. In our meta-analysis, we only included studies in which we defined LRT samples as either BAL or NBL. Furthermore, LRT PCR together with serum and BAL GM were used to supplant the diagnosis of CAPA over colonization (Table 1). The pathophysiology in which patients develop CAPA is not clear. The damage to the respiratory epithelial lining directly by SARS-CoV-2 or as a consequence of the pro-inflammatory cytokine storm phase will lead to mucociliary clearance dysfunction that facilitates the attachment of Aspergillus spores and subsequent invasion [25]. Moreover, COVID-19-induced ARDS, hypoxia, and sepsis may trigger immune paralysis of both the innate and adaptive immune systems, further promoting invasive pulmonary aspergillosis [3, 26, 27].

Recent and prolonged use of corticosteroids within two months at doses greater than $0.3 \mathrm{mg} / \mathrm{kg}$ for three weeks and more are recognized host factors predisposing to invasive pulmonary aspergillosis, as per the EORTC/MSG and AspICU criteria [10, 28]. However, many studies included did not specify the actual doses or total duration of corticosteroids received on a long-term basis by COVID-19 patients. Corticosteroid, in particular dexamethasone, has emerged as the cornerstone therapy for treating COVID-19 patients with acute respiratory failure [29, 30]. No association was observed in critically ill COVID-19 patients who were diagnosed with CAPA than those without CAPA when treated with high-dose corticosteroids in our meta-analysis (Table 2). In critically ill non-COVID-19 patients, stress doses of hydrocortisone are not associated with a high risk of invasive pulmonary aspergillosis [28]. However, concerns exist that even a short course of corticosteroid may predispose to the development of CAPA. Several randomized controlled trials (RCTs) assessing the benefits of corticosteroids had excluded COVID-19 patients at risk or in whom secondary infections were suspected [29, 31]. In those RCTs that evaluated the risk of secondary infections involving ventilator-associated pneumonia, bacteremia, and catheter-related bloodstream or urinary tract infections, no difference was demonstrated in COVID-19 patients receiving high doses of corticosteroids for 14 days or until ICU discharged [32, 33]. Nevertheless, further studies are required to confirm these findings as most studies included were conducted during the first wave of the COVID-19 pandemic before the publication of multiple RCTs demonstrating the benefits of corticosteroids, which explains the inconsistent and infrequent use of corticosteroids in the treatment of COVID-19 patients (Table 2).

Additionally, COPD was identified as a risk factor for developing CAPA in COVID-19 patients (Table 2). This is likely due to the severity of underlying COPD instead of long-term use of inhalational corticosteroids [28, 34]. Aspergillus spores are typically cleared from the lungs by the ciliary action of the bronchial epithelium, but similarly to COVID-19, the ciliary activity in COPD patients is often impaired and inflamed from tobacco smoke, multiple episodes of infection, and repeated epithelial destruction. This allows Aspergillus spores to bind to the epithelial layer and invade the bronchial mucosa and pulmonary parenchyma, eventually the vasculature, and cause pulmonary infarction [35]. Conversely, several studies observed that comorbidities of COPD and diabetes alone were not associated with invasive pulmonary aspergillosis diagnosis in critically ill nonCOVID-19 patients [28]. The risk of developing invasive pulmonary aspergillosis in COPD patients is hypothesized to be from underlying long-term use of high-dose corticosteroid, over $700 \mathrm{mg}$ within three months that impairs immune function, especially macrophages and T-cell activity, responsible for monocyte-mediated damage to Aspergillus spores and hyphae, and promote in-vitro growth of Aspergillus species [34-36]. In our meta-analysis, the number of studies assessing the association of COPD and long-term corticosteroid treatment was limited to three $[14,15,18]$ and seven studies[14-20], respectively. However, a "two-hit" hypothesis might exist in which COPD and long-term use of corticosteroid treatment, especially in high doses, synergistically predispose to CAPA.

According to EORTC/MSG and AspICU criteria, other host factors that predispose to invasive pulmonary aspergillosis development are hematological malignancy, but 
Study or Subgroup Events Total Events Total Weight $\mathrm{M-H}$, Random, 95\% C

1.3.1 Initial Antibiotic Treatment

Delliere et al.

Lahmer et al.

Segrelles-Calvo et al.

$\begin{array}{lllll}16 & 21 & 74 & 87 & 38.8 \%\end{array}$

Vegrez Pintado et al.

wang et al.

Subtotal $(95 \% \mathrm{Cl})$

Total events

$\begin{array}{rr}11 & 11 \\ 7 & 7 \\ 6 & 16\end{array}$

2121

$194208 \quad 7.5 \%$

$\begin{array}{lll}56 & 67 \quad 32.3 \%\end{array}$

$22.5 \%$

391

Heierogeneity. $\mathrm{Tau}^{2}=0.09 ; \mathrm{Chi}^{2}=3.42, \mathrm{df}=3\left\langle\mathrm{P}=0.33 \mathrm{l} ; \mathrm{l}^{2}=12 \%\right.$

Test for overall effect: $Z=0.32(P=0.75)$

1.3.2 Initial Corticosteroid Treatment

$\begin{array}{lllll}18 & 30 & 34 & 78 & 30.5 \%\end{array}$

$\begin{array}{lllllll} & 4 & 7 & 187 & 208 & 23.5 \%\end{array}$

$\begin{array}{lllllll}\text { Velez Pintado et al. } & 2 & 16 & 22 & 67 & 23.4 \%\end{array}$

Wang et al.

Subtotal $(95 \% \mathrm{Cl})$

$\begin{array}{rrrrr}2 & 16 & 22 & 67 & 23.4 \% \\ 6 & 8 & 57 & 96 & 22.6 \%\end{array}$

61

Total events $30 \quad 300$

Heterogeneity. $\mathrm{Tau}^{2}=1.28 ; \mathrm{Chi}^{2}=11.06, \mathrm{df}=3(\mathrm{P}=0.01) ; \mathrm{I}^{2}=73 \%$

Test for overall effect: $Z=0.55(P=0.58)$

\subsubsection{Tocilizumab}

\section{Bartoletti et al.}

Delliere et al.

Segrelles-Calvo et al.

Velez Pintado et al.

Subtotal $(95 \% \mathrm{Cl})$

Total events

$\begin{array}{rrrrr}22 & 30 & 57 & 78 & 42.0 \% \\ 2 & 21 & 2 & 87 & 12.3 \% \\ 5 & 7 & 69 & 208 & 17.3 \% \\ 12 & 16 & 43 & 67 & 28.4 \% \\ & 74 & & 440 & 100.0 \%\end{array}$

$1.01[0.39,2.62]$

$1.94[0.82,4.57)$

$0.15[0.03,0.72]$

$0.28(0.06,1.40)$

$2.05[0.39,10.70]$
$0.69[0.19,2.58]$

Heterogeneity. $\mathrm{Tau}^{2}=0.11 ; \mathrm{Chi}^{2}=3.65, \mathrm{df}=3(\mathrm{P}=0.30) ; \mathrm{I}^{2}=18 \%$

Test for overall effect: $z=1.63(P=0.10)$

1.3.4 Hydroxychloroquine

Bartoletti et al.

Delliere et al.

Segrelles-Calvo et al.

Velez Pintado et al.

Subtotal $(95 \% \mathrm{Cl})$

Total events

$\begin{array}{rrrrr}28 & 30 & 73 & 78 & 24.0 \% \\ 7 & 21 & 27 & 87 & 27.7 \% \\ 3 & 7 & 203 & 208 & 23.8 \% \\ 14 & 16 & 56 & 67 & 24.5 \% \\ & 74 & & 440 & 100.0 \%\end{array}$

$4.47 \mid 0.59,33.791$

$5.04[0.95,26.62]$

$1.67[0.49,5.77]$

$1.85[0.88,3.89]$

Heterogeneity. Tau $^{2}=2.90 ; \mathrm{Chi}^{2}=18.41$, $\mathrm{df}=3(\mathrm{P}=0.0004) ; \mathrm{I}^{2}=84 \%$

Test for overall effect: $Z=0.91(P=0.36)$

1.3.5 Mortality

Bartoletti et al.

Delliere et al.

Cangneux et al.

Lahmer et al.

Segrelles-Calvo et al.

Van Biesen et al.

Velez Pintado et al.

Subtotal $(95 \% \mathrm{Cl})$

Total events

$\begin{array}{rrrrr}13 & 30 & 15 & 78 & 35.5 \% \\ 15 & 21 & 32 & 87 & 27.4 \% \\ 2 & 7 & 4 & 30 & 7.8 \% \\ 4 & 11 & 2 & 21 & 8.2 \% \\ 6 & 7 & 77 & 208 & 6.5 \% \\ 2 & 9 & 5 & 33 & 8.8 \% \\ 1 & 16 & 4 & 67 & 5.8 \% \\ & 101 & & 524 & 100.0 \%\end{array}$

$3.21[1.29,8.02]$

$4.30[1.52,12.18]$

$2.60[0.37,18.25]$

$5.43(0.81,36.51)$

$10.21[1.21,86.38]$

$1.60[0.25,10.05]$

$1.0510 .11,10.091$

$3.39[1.97,5.86]$

Heterogeneity. Tau $^{2}=0.00 ; \mathrm{Chi}^{2}=3.23, \mathrm{di}=6(P=0.78) ; 1^{2}=0 \%$

Test for overall effect: $Z=4.39(P<0.0001)$

1.3.6 RRT

Bartoletti et al

Delliere et al.

Lahmer et al.

Wang et al.

Subtotal $(95 \% \mathrm{Cl})$

Total events

$1.1110 .40,3.061$

$0210.00,0.11)$

$0.38[0.27,6.92]$
$0.07,2.68]$

Odds Ratio

$\mathrm{M}-\mathrm{H}$, Random, 95\% Cl

Heterogeneity. $\mathrm{Tau}^{2}=0.32 \cdot \mathrm{Chi}^{2}=5.15$ df $=3(P=0.16) ; \mathrm{I}^{2}=42 \%$

Test for overall effect: $Z=1.86(P=0.06)$

1.3.7 Inotropic Support

Bartoletti et al.

Delliere et al.

Lahmer et al.

Subtotal $(95 \% \mathrm{Cl})$

Total events

$\begin{array}{rrrrr}11 & 30 & 20 & 78 & 37.9 \% \\ 8 & 21 & 30 & 87 & 35.1 \% \\ 6 & 11 & 3 & 21 & 18.8 \% \\ 1 & 8 & 1 & 96 & 8.2 \%\end{array}$

$1.68(0.68,4.13)$

$1.17[0.44,3.13]$

$7.20[1.31,39.56)$

$3.57[0.76,240.83]$

$2.30[0.95,5.57]$

Heterogeneity. $\mathrm{Tau}^{2}=0.00 ; \mathrm{Chi}^{2}=0.83, \mathrm{di}=1(\mathrm{P}=0.34) ; \mathrm{I}^{2}=0 \%$

Test for overall effect: $Z=0.45(P=0.65)$

$\begin{array}{rrrrrr}19 & 30 & 50 & 78 & 75.8 \% & 0.97[0.40,2.32] \\ 19 & 21 & 70 & 87 & 24.2 \% & 2.31[0.49,10.87] \\ 11 & 11 & 21 & 21 & & \text { Not estimable } \\ & 62 & & 186 & 100.0 \% & 1.19[0.56,2.56]\end{array}$

Not estimable

$[0.56,2.56]$

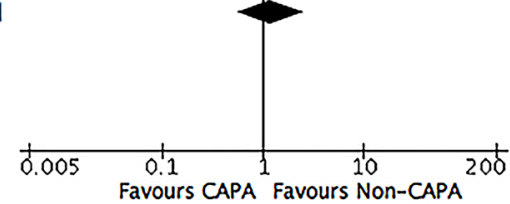


४Fig. 4 Forrest plot of COVID-19 patients divided into CAPA versus non-CAPA. Risk factors of initial COVID-19 therapies (antibiotic, corticosteroid, tocilizumab, and hydroxychloroquine, and outcomes of all-cause in-hospital mortality, inotropic support, and RRT were assessed. The odds ratio was calculated by the Mantel-Haenszel method with a random-effects model. $C I$ confidence intervals, $d f$ degree of freedom, $M-H$ Mantel-Haenszel, $R R T$ renal replacement therapy

not solid tumors, and the recent use within three months of immunosuppressants, specifically those that suppressed $\mathrm{T}$ and $\mathrm{B}$ cells $[10,28]$. In our meta-analysis, no association between the risk of developing CAPA in COVID-19 patients among those with a history of cancers, although the type of cancers, whether solid or hematologic, was not described in the majority of studies included (Table 2). Moreover, we demonstrated no association between the long-term use of immunosuppressants or COVID-19 therapies of hydroxychloroquine and tocilizumab in developing CAPA diagnosis. Tocilizumab, a recombinant humanized monoclonal antibody against interleukin-6 (IL-6) receptor, has been shown to predispose to invasive fungal infections in animal studies [37]. An early observational study involving 544 COVID-19 patients revealed an increased risk of secondary infections (13 vs. 4\%; $P<0.01$ ) in those receiving tocilizumab [38]. Conversely, a large retrospective study of 4,485 COVID19 patients demonstrated no association in the frequency of developing secondary infections among those receiving tocilizumab therapy [39]. Similar findings were noted in a recent RCT of 389 COVID-19 patients comparing tocilizumab with placebo [40]. Nevertheless, a large, well-designed observational study that focuses explicitly on the type of immunosuppressants, dosing, and duration of administration with the risk of developing CAPA is required to confirm these findings.

Critically ill non-COVD-19 patients diagnosed with invasive pulmonary aspergillosis have a higher SOFA score with greater requirement of IMV (100 vs. $82.4 \%$; $P<0.01)$, RRT (44.3 vs. $23.0 \% ; P<0.01$ ), and higher death rate $(39.5$ vs. $23.6 \% ; P<0.01)$ despite antifungal therapy [28]. Similar findings were noted in our meta-analysis where many critically ill COVID-19 patients with CAPA were mechanically ventilated and had greater disease severity based on their initial SOFA score, quicker time to ICU admission from illness onset, and higher all-cause in-hospital mortality rate than those without CAPA (Table 2). However, the requirement of inotropic support, RRT, and duration of IMV were similar. Compared to influenza patients, a retrospective multi-center cohort study of 81 influenza patients observed an increase in morbidity and mortality among those with invasive pulmonary aspergillosis diagnosis with prolonged hospital LOS and higher requirement of IMV, vasopressors, RRT, and extracorporeal membrane oxygenation, despite receiving antifungal therapy [41]. A larger retrospective multi-center cohort study of 432 influenza patients revealed that those diagnosed with invasive pulmonary aspergillosis were more likely to have comorbidities of diabetes, hematological malignancy, neutropenia, solid organ transplant, exposure to high-dose prolonged corticosteroids before admission, and greater severity of illness with longer ICU LOS, the higher requirement of vasopressors and RRT with poor mortality rate [8]. The lack of awareness of invasive pulmonary aspergillosis in influenza patients resulting in the delayed initiation of antifungal therapy is an independent risk factor for increased mortality [42].

Our meta-analysis's strength is that it is the only metaanalysis that assesses the risk factors of developing CAPA and associated outcomes in COVID-19 patients using highquality studies with comparative data (Tables 2 and 3). We employed stringent inclusion and exclusion criteria while using recognized invasive pulmonary aspergillus classifications and accepting only BAL and NBL as LRT specimens. Multiple studies from various countries involving COVID19 patients of different ethnicities (Table 1) ensured that the pragmatic results of our study are relevant and represent all populations. We excluded patients with non-Aspergillus microorganisms identified on LRT specimens as we recognized that non-Aspergillus microorganisms-associated pneumonia, which is common in COVID-19 patients, especially in those requiring mechanical ventilation, are associated with higher morbidity and mortality that might confound the outcomes of our meta-analysis [43]. Furthermore, distinguishing between CAPA and non-Aspergillus-associated pneumonia in COVID-19 patients are challenging due to the overlapping clinical and radiological features [44, 45]. The pathophysiology of COVID-19 in the development of lung injury and the role played in enhancing ongoing infection need to be investigated in further studies. The low heterogeneity among studies describing the in-hospital mortality in CAPA patients prevents underestimating ICU LOS, IMV duration, and the need for inotropic and RRT (Table 2). The funnel plot demonstrated symmetry in patients' clinical characteristics, indicating a low likelihood of publication bias (Fig. 5).

There are several limitations to our meta-analysis. The heterogeneity among studies with a varying incidence of CAPA between 3.3 and $34.4 \%$ and clinical characteristics (Tables 1 and 2) described may limit the ability to perform an accurate meta-analysis. This could be attributed to the difference in clinical and mycological evidence varied in clinical centers from different parts of the world, lack of clinical awareness, and standardized diagnostic approach for evaluating CAPA, particularly when clinical features and radiological findings in CAPA resemble those of severe COVID-19 pneumonia [10, 14, 46]. The use of the randomeffects model as a solution towards clinical heterogeneity in results may have resulted in a more conservative treatment 
Fig. 5 Funnel plot

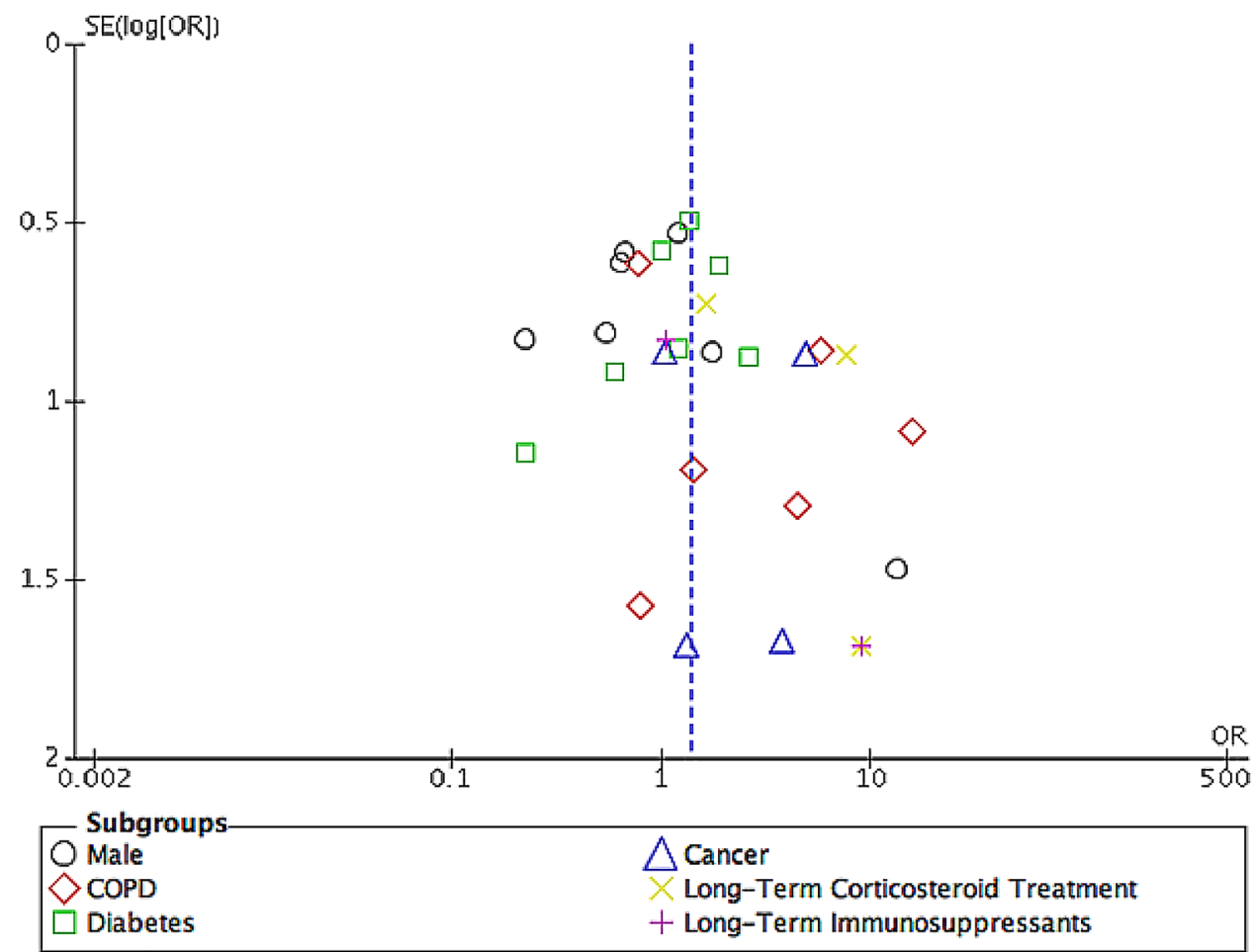

effect and wider CIs. Furthermore, the reluctance to perform aerosol-generating procedures of bronchoscopies in critically ill COVID-19 patients is due to concerns of transmission and the risk of triggering hemodynamic instability that limits respiratory tract samples to sputum and TA [46]. These likely explain the low number of studies available in the current literature. Although TAs are potentially safer alternative investigative options, they cannot effectively distinguish between colonization and true infection, leading to overdiagnosis of CAPA and unnecessary treatments. The low yield of serum GM will further complicate the screening process for invasive pulmonary aspergillosis in COVID-19 patients due to its poor sensitivity at $21 \%$, especially in nonimmunocompromised patients [46]. Despite BAL GM being a superior diagnostic test to confirm CAPA, as an aerosolgenerating procedure, it is rarely performed. Certain studies even reported that the variability in diagnostic criteria used for CAPA diagnosis might lead to overdiagnosis, overtreatment, and overestimating the true risk factors and associated outcomes [14, 16, 22]. Therefore, significant predictors and prognostic factors associated with outcomes may have been missed due to a lack of analyzable data. Autopsy findings among 677 deceased COVID-19 patients during the first wave of pandemic demonstrated that the incidence of tissue-proven invasive fungal diseases was up to $2 \%$ [47]. Important environmental factors such as temporary facilities/medical centers that were rapidly assembled in many countries to accommodate the increase in healthcare capacity during the pandemic that does not adhere to rigorous ventilation protocols present within permanent hospitals might contribute to the spread of COVID-19 among the critically ill patients from increased exposure to dust and ambient air containing Aspergillus spores [48]. In general, the duration of patient enrollment and follow-up for all the included studies (Table 1) were performed during the first wave of the COVID-19 pandemic and might not accurately represent the actual risk factors and outcomes due to the ever-evolving improvement in the management and treatment of COVID-19 patients.

\section{Conclusion}

COVID-19 patients diagnosed with CAPA are likely to be older with underlying COPD. Long-term use of corticosteroids may predispose to CAPA. Patients with CAPA diagnosis have more significant disease severity based on SOFA scores, earlier onset of ICU admission, and higher allcause in-hospital mortality rate than those without CAPA. Nevertheless, the results of our meta-analysis represent the patient population during the first wave of the COVID-19 pandemic, and better studies are required with the advancement of COVID-19 therapies to determine if the same risk factors and outcomes are observed during the second wave of the pandemic and beyond. Although early CAPA diagnosis likely improves the morbidity and all-cause in-hospital mortality in COVID-19 patients, the benefit of timely initiation of appropriate antifungals is unclear. Furthermore, 
whether antifungal prophylaxis is superior to a standardized diagnostic approach combined with prompt initiation of antifungal therapy for managing CAPA patients remains to be examined. In the absence of antifungal prophylaxis, adequate screening for CAPA, recognition of its risk factors, and exclusion of colonization are essential.

Author contributions WHC: conceptualization, methodology, investigation, data curation, writing - original draft; BKS: conceptualization, methodology, investigation, data curation, writing - review and editing; KPN: conceptualization, methodology, investigation, data curation, supervision, project administration, writing—review and editing.

\section{Funding None.}

\section{Declarations}

Conflict of interest We declare no conflicts of interest.

Ethical approval Ethical approval and informed consent were not required for this study as it was a systematic review of previously published studies. The protocol for this review was registered and published in the International Prospective Register of Systematic Reviews (PROSPERO) under reference number CRD42021247177.

\section{References}

1. Chong WH, Saha BK, Ramani A, Chopra A. State-of-theart review of secondary pulmonary infections in patients with COVID-19 pneumonia. Infection. 2021;49:591-605.

2. Zhou F, Yu T, Du R, Fan G, Liu Y, Liu Z, et al. Clinical course and risk factors for mortality of adult inpatients with COVID19 in Wuhan, China: a retrospective cohort study. Lancet. 2020;395:1054-62.

3. Arastehfar A, Carvalho A, van de Veerdonk FL, Jenks JD, Koehler $\mathrm{P}$, Krause R, et al. COVID-19 associated pulmonary aspergillosis (CAPA) - from immunology to treatment. J Fungi. 2020;6:91.

4. Armstrong-James D, Youngs J, Bicanic T, Abdolrasouli A, Denning DW, Johnson E, et al. Confronting and mitigating the risk of COVID-19 associated pulmonary aspergillosis (CAPA). Eur Respir J. 2020;23:2002554.

5. Koehler P, Bassetti M, Chakrabarti A, Chen SCA, Colombo AL, Hoenigl M, et al. Defining and managing COVID-19-associated pulmonary aspergillosis: the 2020 ECMM/ISHAM consensus criteria for research and clinical guidance. Lancet Infect Dis. 2021;21:e149-62.

6. Chong WH, Ibrahim A, Saha BK. The evolution of invasive pulmonary aspergillosis on chest imaging in response to antifungal therapy. BMJ Case Rep. 2021;14:e242576.

7. Chong WH, Neu KP. Incidence, diagnosis and outcomes of COVID-19-associated pulmonary aspergillosis (CAPA): a systematic review. J Hosp Infect. 2021;113:115-29.

8. Schauwvlieghe AFAD, Rijnders BJA, Philips N, Verwijs R, Vanderbeke L, Van Tienen $\mathrm{C}$, et al. Invasive aspergillosis in patients admitted to the intensive care unit with severe influenza: a retrospective cohort study. Lancet Respir Med. 2018;6:782-92.

9. Verweij PE, Rijnders BJA, Brüggemann RJM, Azoulay E, Bassetti M, Blot S, et al. Review of influenza-associated pulmonary aspergillosis in ICU patients and proposal for a case definition: an expert opinion. Intensive Care Med. 2020;46:1524-35.
10. Donnelly JP, Chen SC, Kauffman CA, Steinbach WJ, Baddley JW, Verweij PE, et al. Revision and update of the consensus definitions of invasive fungal disease from the European organization for research and treatment of cancer and the mycoses study group education and research consortium. Clin Infect Dis. 2020;71:1367-76.

11. Stang A. Critical evaluation of the Newcastle-Ottawa scale for the assessment of the quality of nonrandomized studies in metaanalyses. Eur J Epidemiol. 2010;25:603-5.

12. DerSimonian R, Laird N. Meta-analysis in clinical trials. Control Clin Trials. 1986;7:177-88.

13. Higgins JPT, Thompson SG, Deeks JJ, Altman DG. Measuring inconsistency in meta-analyses. BMJ. 2003;327:557-60.

14. Bartoletti M, Pascale R, Cricca M, Rinaldi M, Maccaro A, Bussini L, et al. Epidemiology of invasive pulmonary aspergillosis among COVID-19 intubated patients: a prospective study. Clin Infect Dis. 2020; ciaa1065.

15. Dellière S, Dudoignon E, Fodil S, Voicu S, Collet M, Oillic P-A, et al. Risk factors associated with COVID-19-associated pulmonary aspergillosis in ICU patients: a French multicentric retrospective cohort. Clin Microbiol Infect. 2020; S1198-743X(20)30756-4.

16. Gangneux J-P, Reizine F, Guegan H, Pinceaux K, Le Balch P, Prat E, et al. Is the COVID-19 pandemic a good time to include Aspergillus molecular detection to categorize aspergillosis in ICU patients? A monocentric experience. J Fungi. 2020;6:105.

17. Lahmer T, Kriescher S, Herner A, Rothe K, Spinner CD, Schneider J, et al. Invasive pulmonary aspergillosis in critically ill patients with severe COVID-19 pneumonia: results from the prospective AspCOVID-19 study. PLoS One. 2021; 16:e0238825.

18. Van Biesen S, Kwa D, Bosman RJ, Juffermans NP. Detection of invasive pulmonary aspergillosis in COVID-19 with nondirected BAL. Am J Respir Crit Care Med. 2020;202:1171-3.

19. Vélez Pintado M, Camiro-Zúñiga A, Aguilar Soto M, Cuenca D, Mercado M, Crabtree-Ramirez B, et al. COVID-19-associated invasive pulmonary aspergillosis in a tertiary care center in Mexico City. Med Mycol. 2021;59:828-33.

20. Wang J, Yang Q, Zhang P, Sheng J, Zhou J, Qu T. Clinical characteristics of invasive pulmonary aspergillosis in patients with COVID-19 in Zhejiang, China: a retrospective case series. Crit Care. 2020;24:299.

21. Segrelles-Calvo G, Araújo GRS, Llopis-Pastor E, Carrillo J, Hernández-Hernández M, Rey L, et al. Prevalence of opportunistic invasive aspergillosis in COVID-19 patients with severe pneumonia. Mycoses. 2021;64:144-51.

22. van Grootveld R, van Paassen J, de Boer MGJ, Claas ECJ, Kuijper EJ, van der Beek MT, et al. Systematic screening for COVID-19 associated invasive aspergillosis in ICU patients by culture and PCR on tracheal aspirate. Mycoses. 2021;64:641-50.

23. Contou D, Dorison M, Rosman J, Schlemmer F, Gibelin A, Foulet $\mathrm{F}$, et al. Aspergillus-positive lower respiratory tract samples in patients with the acute respiratory distress syndrome: a 10-year retrospective study. Ann Intensive Care. 2016;6:52.

24. Fekkar A, Lampros A, Mayaux J, Poignon C, Demeret S, Constantin J-M, et al. Occurrence of Invasive Pulmonary Fungal Infections in Patients with Severe COVID-19 Admitted to the ICU. Am J Respir Crit Care Med. 2021;203:307-17.

25. Apostolopoulou A, Esquer Garrigos Z, Vijayvargiya P, Lerner $\mathrm{AH}$, Farmakiotis D. Invasive pulmonary aspergillosis in patients with SARS-CoV-2 infection: a systematic review of the literature. Diagnostics. 2020;10:807.

26. Fliesser M, Wallstein M, Kurzai O, Einsele H, Löffler J. Hypoxia attenuates anti-Aspergillus fumigatus immune responses initiated by human dendritic cells. Mycoses. 2016;59:503-8. 
27. Hotchkiss RS, Monneret G, Payen D. Sepsis-induced immunosuppression: from cellular dysfunctions to immunotherapy. Nat Rev Immunol. 2013;13:862-74.

28. Blot SI, Taccone FS, Van den Abeele A-M, Bulpa P, Meersseman $\mathrm{W}$, Brusselaers $\mathrm{N}$, et al. A clinical algorithm to diagnose invasive pulmonary aspergillosis in critically Ill patients. Am J Respir Crit Care Med. 2012;186:56-64.

29. RECOVERY Collaborative Group, Horby P, Lim WS, Emberson JR, Mafham M, Bell JL, et al. Dexamethasone in Hospitalized Patients with Covid-19. N Engl J Med. 2021;38:693-704.

30. The WHO Rapid Evidence Appraisal for COVID-19 Therapies (REACT) Working Group, Sterne JAC, Murthy S, Diaz JV, Slutsky AS, Villar J, et al. Association between administration of systemic corticosteroids and mortality among critically Ill patients with COVID-19: a meta-analysis. JAMA. 2020;324:1330.

31. Petersen MW, Meyhoff TS, Helleberg M, Kjær MN, Granholm A, Hjorts $\varnothing$ CJS, et al. Low-dose hydrocortisone in patients with COVID-19 and severe hypoxia (COVID STEROID) trial-protocol and statistical analysis plan. Acta Anaesthesiol Scand. 2020;64:1365-75.

32. Dequin P-F, Heming N, Meziani F, Plantefève G, Voiriot G, Badié $\mathrm{J}$, et al. Effect of hydrocortisone on 21-day mortality or respiratory support among critically Ill patients with COVID-19: a randomized clinical trial. JAMA. 2020;324:1298.

33. Tomazini BM, Maia IS, Cavalcanti AB, Berwanger O, Rosa RG, Veiga VC, et al. Effect of dexamethasone on days alive and ventilator-free in patients with moderate or severe acute respiratory distress syndrome and COVID-19: the CoDEX randomized clinical trial. JAMA. 2020;324:1307.

34. Tutar N, Metan G, Koç AN, Yilmaz I, Bozkurt I, Simsek ZO, et al. Invasive pulmonary aspergillosis in patients with chronic obstructive pulmonary disease. Multidiscip Respir Med. 2013;8:59.

35. Bulpa P, Dive A, Sibille Y. Invasive pulmonary aspergillosis in patients with chronic obstructive pulmonary disease. Eur Respir J. 2007;30:782-800.

36. Ader F, Nseir S, Le Berre R, Leroy S, Tillie-Leblond I, Marquette $\mathrm{CH}$, et al. Invasive pulmonary aspergillosis in chronic obstructive pulmonary disease: an emerging fungal pathogen. Clin Microbiol Infect. 2005;11:427-9.

37. Cenci E, Mencacci A, Casagrande A, Mosci P, Bistoni F, Romani L. Impaired antifungal effector activity but not inflammatory cell recruitment in interleukin-6-deficient mice with invasive pulmonary aspergillosis. J Infect Dis. 2001;184:610-7.

38. Guaraldi G, Meschiari M, Cozzi-Lepri A, Milic J, Tonelli R, Menozzi M, et al. Tocilizumab in patients with severe COVID-19: a retrospective cohort study. Lancet Rheumatol. 2020;2:e474-84.

39. Gupta S, Wang W, Hayek SS, Chan L, Mathews KS, Melamed ML, et al. Association between early treatment with tocilizumab and mortality among critically Ill patients with COVID-19. JAMA Intern Med. 2021;181:41-51.

40. Salama C, Han J, Yau L, Reiss WG, Kramer B, Neidhart JD, et al. Tocilizumab in patients hospitalized with COVID-19 pneumonia. N Engl J Med. 2021;384:20-30.

41. Waldeck F, Boroli F, Suh N, Wendel Garcia PD, Flury D, Notter J, et al. Influenza-associated aspergillosis in critically-ill patients-a retrospective bicentric cohort study. Eur J Clin Microbiol Infect Dis. 2020;39:1915-23.

42. van de Veerdonk FL, Kolwijck E, Lestrade PPA, Hodiamont CJ, Rijnders BJA, van Paassen J, et al. Influenza-associated aspergillosis in critically Ill patients. Am J Respir Crit Care Med. 2017;196:524-7.

43. Maes M, Higginson E, Pereira-Dias J, Curran MD, Parmar S, Khokhar F, et al. Ventilator-associated pneumonia in critically ill patients with COVID-19. Crit Care Lond Engl. 2021;25:25.

44. Ripa M, Galli L, Poli A, Oltolini C, Spagnuolo V, Mastrangelo A, et al. Secondary infections in patients hospitalized with COVID19: incidence and predictive factors. Clin Microbiol Infect Off Publ Eur Soc Clin Microbiol Infect Dis. 2021;27:451-7.

45. Chong WH, Chieng H, Tiwari A, Beegle S, Feustel PJ, Ghalib S, et al. Incidence and risk factors for secondary pulmonary infections in patients hospitalized with coronavirus disease 2019 pneumonia. Am J Med Sci. 2021;S0002-9629:00137-43.

46. Hoenigl M. Invasive Fungal Disease Complicating Coronavirus Disease 2019: When It Rains, It Spores. Clinical Infectious Diseases. 2020; ciaa1342.

47. Kula BE, Clancy CJ, Hong Nguyen M, Schwartz IS. Invasive mould disease in fatal COVID-19: a systematic review of autopsies. Lancet Microbe. 2021;2:e405-14.

48. Thompson Iii GR, Cornely OA, Pappas PG, Patterson TF, Hoenigl M, Jenks JD, et al. Invasive aspergillosis as an under-recognized superinfection in COVID-19. Open Forum Infect Dis. 2020;7:ofaa242. 